(1)

CrossMark

\title{
Reply: Is high-dose glucocorticoid beneficial in COVID-19?
}

Reply to V. Muthu and co-workers:

In their correspondence, V. Muthu and co-workers raise some issues related to our recent clinical trial "Intravenous methylprednisolone pulse as a treatment for hospitalised severe COVID-19 patients: results from a randomised controlled clinical trial" [1]. This provides a welcome opportunity to further explain the outcome of using a high dose of glucocorticoid for the treatment of patients with severe coronavirus disease 2019 (COVID-19).

$\mathrm{V}$. Muthu and colleagues suggest that our mortality rate was higher than expected based on the RECOVERY trial; however, there are clear differences in the patient populations, which make this an inappropriate comparison. The mortality rate of our control group is more than the mortality rate of the RECOVERY trial because all of the patients included in this study were hospitalised in an intensive care unit (ICU) and all of them received oxygen support. However, in the RECOVERY trial, 6425 hospitalised COVID-19 patients were included (not mentioned at ICU or general units) and $24 \%$ of them did not receive oxygen support [2]. Besides, the mortality rate of COVID-19 is different among different ethnic groups. In an observational study conducted on 905 COVID-19 patients hospitalised in Imam Khomeini hospital complex in Tehran, Iran, the mortality rate of ICU admitted patients was reported to be 50\% [3]. V. Muthu and co-workers also claim that a higher mortality rate in the control arm may be due to the better supportive care provided to the intervention arm. We believe this is an inappropriate suggestion because, according to moral and ethical guidelines, equal supportive care was provided for all patients enrolled in this study, in both standard care and intervention arms.

V. Muthu and co-workers also state that the justification provided for our sample size is inadequate. To address this point we note that, although the beneficial use of corticosteroid treatment in COVID-19 patients who are in the pulmonary phase has now been shown in different clinical studies, the use of corticosteroids for the treatment of COVID-19 was unproven when we conducted this clinical trial (20 April, 2020), and the World Health Organization recommended against the routine use of systemic corticosteroids for the treatment of viral pneumonia [4]. Therefore, for using a high dose of methylprednisolone, the minimum sample size was estimated.

V. Muthu and colleagues express that clinical improvement in our study was defined by quick fever subsidence and a sense of well-being in subjective parameters. We postulated that hospital discharge was performed based on the national discharge protocol for COVID-19 at that time in our trial and the clinical improvement was determined based on oxygen saturation more than $93 \%$, Borg score more than 3 , lack of fever for 3 days, improved dyspnoea, normal urinary output, tolerated oral regimen and reduced C-reactive protein level without any treatment adverse effects. However, we followed up discharged patients in both groups, and the general symptoms, clinical features and characteristics of the recovered patients were also evaluated 1 week after discharge time. The data regarding patients' clinical features 7 days after discharge time were presented in supplementary table S2 of the article [1].

V. Muthu and co-workers also declare that a higher prevalence of diabetes mellitus (DM) in the control group in our study resulted in reduced adverse events in the intervention group. Since the patients in our study were randomly allocated into two groups, we did not match them according to their coexisting conditions. Although the prevalence of DM in the patients of the usual care group was higher, the level of respiratory rate and the level of heart rate were significantly elevated in the methylprednisolone group. Besides, the number of patients who had pulmonary involvement more than $70 \%$, and the number of patients who needed noninvasive ventilation or reservoir mask before enrolment was higher in the

@ERSpublications

Our clinical trial supports the beneficial use of a high dose of methylprednisolone for the treatment of severe COVID-19 patients. https://bit.ly/3qvc4Kz

Cite this article as: Edalatifard M, Akhtari M, Salehi M, et al. Reply: Is high-dose glucocorticoid beneficial in COVID-19? Eur Respir J 2021; 57: 2100324 [https://doi.org/10.1183/13993003.00324-2021]. 
methylprednisolone group. Besides, we did not observe a difference in the level of patients' blood sugar with regard to their clinical status. It is worth mentioning that uncontrolled DM is one of our exclusion criteria and COVID-19 patients with uncontrolled DM were excluded from this study [1]. Regarding the use of high doses of glucocorticoid and an increased risk of infectious complications in patients, we emphasised that we did not see any infectious adverse events in the methylprednisolone group, including COVID-19-associated pulmonary aspergillosis and mucormycosis.

To conclude, we appreciate the interest of V. Muthu and colleagues in our article and appreciate the opportunity to share our experience regarding using glucocorticoids for the treatment of COVID-19 patients. Our data support the beneficial use of a high dose of methylprednisolone for the treatment of severe COVID-19 patients; however, more clinical studies with a higher sample size are needed.

Maryam Edalatifard ${ }^{1}$, Maryam Akhtari $\circledast^{2,3}$, Mohammadreza Salehi ${ }^{4}$, Elham Farhadi ${ }^{2,3}$, Ahmadreza Jamshidi ${ }^{2}$, Mahdi Mahmoudi $\oplus^{2,3}$ and Abdolrahman Rostamian ${ }^{5}$

${ }^{1}$ Advanced Thoracic Research Center, Tehran University of Medical Sciences, Tehran, Iran. ${ }^{2}$ Rheumatology Research Center, Tehran University of Medical Sciences, Tehran, Iran. ${ }^{3}$ Inflammation Research Center, Tehran University of Medical Sciences, Tehran, Iran. ${ }^{4}$ Dept of Infectious and Tropical Medicines, Tehran University of Medical Sciences, Tehran, Iran. ${ }^{5}$ Rheumatology Research Center, Imam Khomeini Hospital, Tehran University of Medical Sciences, Tehran, Iran.

Correspondence: Abdolrahman Rostamian, Rheumatology Research Center, Imam Khomeini Hospital, P.O. BOX: 1418419967, Tehran, Iran. E-mail: arostamian@tums.ac.ir

Received: 2 Feb 2021 | Accepted: 11 Feb 2021

Conflict of interest: None declared.

\section{References}

1 Edalatifard M, Akhtari M, Salehi M, et al. Intravenous methylprednisolone pulse as a treatment for hospitalised severe COVID-19 patients: results from a randomised controlled clinical trial. Eur Respir J 2020; 56: 2002808.

2 RECOVERY Collaborative Group, Horby P, Lim WS, et al. Dexamethasone in hospitalized patients with Covid-19. N Engl J Med 2021; 384: 693-704.

3 Allameh SF, Nemati S, Ghalehtaki R, et al. Clinical characteristics and outcomes of 905 COVID-19 patients admitted to Imam Khomeini Hospital Complex in the Capital City of Tehran, Iran. Arch Iran Med 2020; 23: 766-775.

4 World Health Organization. Clinical Management of COVID-19: living guidance. Geneva, World Health Organization, 2020. www.who.int/publications/i/item/clinical-management-of-covid-19. 